\title{
Conceptual Engineering of Plasma-Focus Thermonuclear Pulsors
}

\author{
César Moreno, Horacio Bruzzone, Javier Martínez, and Alejandro Clausse
}

\begin{abstract}
The basic engineering criteria for conceptual design of plasma focus devices is derived from a thermonuclear model, and applied successfully to the operation of small neutron pulsors. The theory is able to explain the variation of the neutron yield with the gas pressure in deuterium-filled chambers, the current evolution, and the electrode geometrical parameters. The performance of a prototype designed to optimize the flux/fluence ratio is presented, contrasting the experimental outcomes with the model. A set of effective design parameters is deduced, which ensure a band confidence of $20 \%$.
\end{abstract}

Index Terms-Current sheet, fusion, neutrons, plasma-focus, plasma pinches, pressure dependence, thermonuclear, $Z$-pinch.

\section{INTRODUCTION}

$\mathbf{P}$ LASMA neutron pulsors are small thermonuclear devices based on the pinch phenomenon occurring during the path of high electric currents through gases. Essentially, the plasma pinch is a very fast compression and the consequent heating of the plasma by means of the associated magnetic field, during which substantial amounts of different types of radiation are produced, namely, x-rays, electrons, ions, and, using deuterium, neutrons from nuclear reactions. Different types of configurations can be used to induce the pinch formation: plasma focus, $z$-pinch, $\theta$-pinch, among others. To the present, the plasma focus has been the most successful device from the point of view of neutron generation.

Since the pioneer work of Filipov [1] and Mather [2], plasma focus $(\mathrm{PF})$ has been extensively studied by research laboratories around the world, where several devices have been developed over the years aiming to increase the neutron emission [3], [4]. Currently, PF pulsors are among the cheapest available neutron generators, with unique features of extremely short pulses (hundreds of nanoseconds) that suit them for a number of interesting applications [5].

In spite of the accumulated experience in operating plasma pinches over the last $30 \mathrm{yr}$ in a wide range of energies, with remarkable success in producing neutrons, there is still controversy regarding the physical mechanisms involved in the pinch phenomenon. Most of the studies performed in PF pulsors are empirical, and not many theoretical calculations have been done in order to build a base for reliable design models. The numerical analysis reported in the literature [6], [7] are generally related to the plasma acceleration process, whereas little attention

Manuscript received March 7, 2000; revised July 6, 2000.

The authors are with the National Research Network PLADEMA, Interinstitutional Program of Dense Magnetized Plasmas, Tandil, 7000 Argentina (e-mail: clausse@exa.unicen.edu.ar).

Publisher Item Identifier S 0093-3813(00)09977-X.

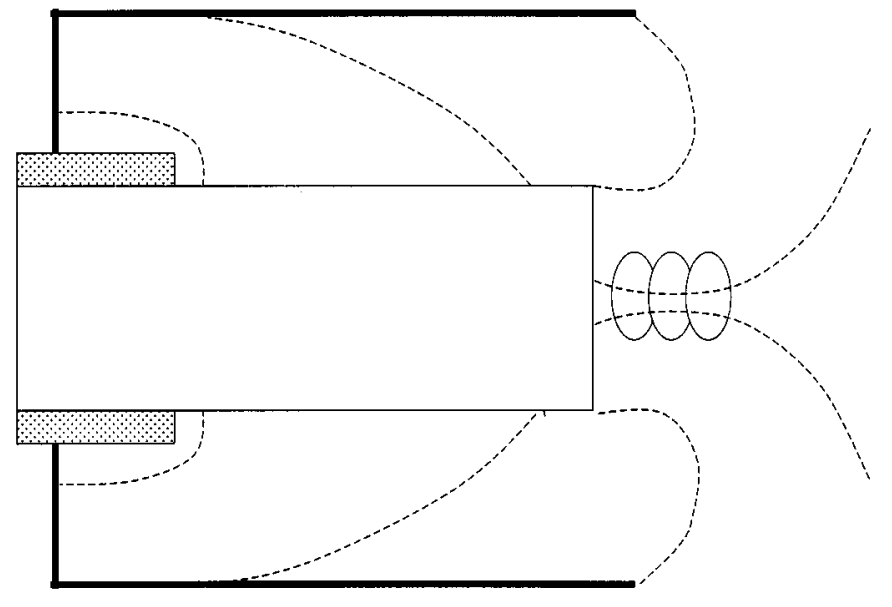

Fig. 1. Diagram of the plasma focus phenomenon.

has been paid to the neutron production and its relation to the current, filling pressure, and other operating conditions.

In this paper, a theoretical and experimental study of the neutron emission in PF pulsors is presented. A conceptual model is produced, which accounts for the features that would be expected if neutrons come exclusively from thermonuclear reactions. The theory is able to explain the relation of the neutron production with the gas-filling pressure, the current evolution, and the geometrical parameters of the device. A set of experiments was designed in order to compare with the model, leading to good agreements. The model also shows good performance against other experiments reported in the past. Finally, a set of design criteria is recommended for the conceptual design of $\mathrm{PF}$ neutron pulsors.

\section{Model of Thermonuclear Plasma-Focus}

Plasma focus is a phenomenon occurring at the open end of coaxial electrodes when an intense electrical discharge between them is induced by external means. Fig. 1 shows a scheme of the system. Two coaxial electrodes are located inside a vacuum chamber filled with deuterium gas at low pressure (neutrons were reported from 0.1 to 10 mbar). A charged capacitor bank is connected to the closed end of the electrodes through a switch (spark-gap). After closing the switch, a gas discharge starts in the gap between the electrodes forming an umbrella-like plasma layer. The azimuthal magnetic field located in the torus enclosed by the current produces a $\vec{J} \times \vec{B}$ force that pushes the sheath toward the open end of the electrodes. The rundown of the current sheet is a sweeping supersonic shock that propagates and collects with certain efficiency $\xi$ the gas particles ahead of the 


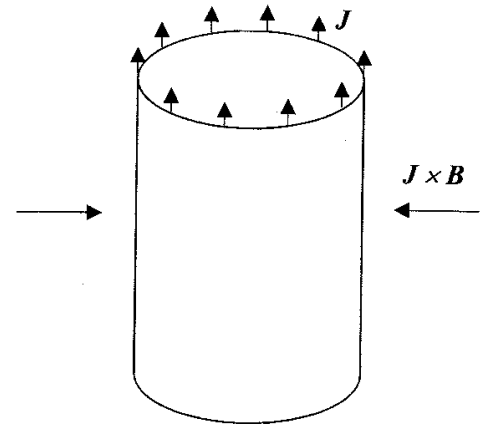

Fig. 2. Ideal $Z$-pinch.

front (snowplow scenario). On its arrival at the open end (some microseconds after triggering), the magnetic field starts to contract, accelerating the plasma toward the axis (runover stage). Finally, the sheath clashes on the axis in the form of a small dense plasma cylinder (focus). The lifetime of the focus is about 300 ns.

\section{A. Radial Plasma Pinch}

Let us consider an ideal cylindrical pinch of autocompressed deuterium plasma (Fig. 2), producing thermonuclear reactions during a short period of time when the density and temperature are sufficiently high. The total yield production during such pulse is given by (see Table I)

$$
Y=\frac{1}{2} \int_{\Delta t} n^{2}\langle\sigma v\rangle V d t
$$

Equation (1) can be calculated provided that the time dependence of the deuteron density $n$, the thermal cross section $\langle\sigma v\rangle$, and the pinch volume $V$ is known. Ideally, the pinch compression can be described as a nonlinear oscillator, where the Lorentz force competes with the kinetic pressure from the gas. Assuming a constant electrical current $I$ flowing axially along an adiabatic plasma cylinder, the radial acceleration is given by

$$
\ddot{r}=\frac{h}{M}\left(\frac{2 \pi p_{R} r_{R}^{2 \gamma}}{r^{2 \gamma-1}}-\frac{\mu_{o} I^{2}}{4 \pi r}\right) .
$$

The parameters $p_{R}$ and $r_{R}$ in (2) are reference values of pressure and radius along an isoentropic transformation. The radius $r_{B}$ for which the right-hand side of (2) vanishes (Bennett equilibrium) is given by

$$
r_{B}^{2(\gamma-1)}=\frac{8 \pi^{2} p_{R} r_{R}^{2 \gamma}}{\mu_{o} I^{2}}
$$

The corresponding Bennett temperature $T_{B}$ is

$$
T_{B}=\frac{h}{k} \frac{\mu_{o}}{16 \pi} \frac{I^{2}}{N}
$$

A useful assessment of the integral in (1) can be produced by assuming that during the reactive period the variables remain close to the Bennett equilibrium. Using this assumption, (1) becomes

$$
Y=\frac{N^{2}\langle\sigma v\rangle_{B} \Delta t}{2 V_{B}}
$$

\begin{tabular}{|c|c|c|}
\hline Symbol & Parameter & Unit \\
\hline$\vec{C}$ & Capacity & {$[\mathrm{F}]$} \\
\hline$h$ & Pinch length & [m] \\
\hline I & Pinch current & [A] \\
\hline$k$ & Boltzmann constant, $1.3810^{-23} \mathrm{~J} /{ }^{\circ} \mathrm{K}$ & {$\left[\mathrm{J} /{ }^{\circ} \mathrm{K}\right]$} \\
\hline$l$ & Inductance per unit length, $\left(\mu_{o} / 2 \pi\right) \ln \left(r_{2} / r_{1}\right)$ & {$\left[\mathrm{J} / \mathrm{mA}^{2}\right]$} \\
\hline$L$ & External inductance & {$[\mathrm{Hr}]$} \\
\hline$m_{i}$ & Deuteron mass, $3.3410^{-27} \mathrm{~kg}$ & {$[\mathrm{~kg}]$} \\
\hline$m$ & Collected mass per unit length & {$[\mathrm{kg} / \mathrm{m}]$} \\
\hline$M$ & Pinch mass, $N m_{i}$ & [kg] \\
\hline$n$ & Deuteron number density & {$\left[\mathrm{m}^{-3}\right]$} \\
\hline$N$ & Number of deuterons in the pinch volume & {$[-]$} \\
\hline$p_{o}$ & Filling pressure & {$\left[\mathrm{N} / \mathrm{m}^{2}\right]$} \\
\hline$Q$ & Electrical charge & {$[\mathrm{C}]$} \\
\hline$Q_{o}$ & Initial electrical charge & {$[\mathrm{C}]$} \\
\hline$r$ & Pinch radius & [m] \\
\hline$r_{I}$ & External electrode radius & [m] \\
\hline$r_{2}$ & Internal electrode radius & [m] \\
\hline$r_{B}$ & Bennett radius & [m] \\
\hline$r_{s}$ & Isoentropic reference & [m] \\
\hline$t$ & Time & [s] \\
\hline$T$ & Effective pinch temperature & {$\left[{ }^{\circ} \mathrm{K}\right]$} \\
\hline$T_{o}$ & Stagnant gas temperature & {$\left[{ }^{\circ} \mathrm{K}\right]$} \\
\hline$T_{B}$ & Effective Bennett pinch temperature & {$\left[{ }^{\circ} \mathrm{K}\right]$} \\
\hline$T^{*}$ & Cross section parameter, $7.6510^{100} \mathrm{~K}$ & {$\left[{ }^{\circ} \mathrm{K}\right]$} \\
\hline$V$ & Pinch volume, $V=\pi r^{2} h$ & {$\left[\mathrm{~m}^{3}\right]$} \\
\hline$V_{B}$ & Bennett pinch volume & {$\left[\mathrm{m}^{3}\right]$} \\
\hline$x$ & Axial coordinate & [m] \\
\hline$Y$ & Neutron yield & {$[-]$} \\
\hline$z$ & Anode length & [m] \\
\hline$\Delta t$ & Pinch duration & {$[\mathrm{s}]$} \\
\hline$\varepsilon$ & Expansion parameter & {$[-]$} \\
\hline$\gamma$ & Specific heat ratio, $5 / 3$ & {$[-]$} \\
\hline$\theta$ & Dimensionless temperature, $T / T^{*}$ & {$[-]$} \\
\hline$\theta_{B}^{*}$ & Critical dimensionless temperature, $(4 / 29)^{3}$ & {$[-]$} \\
\hline$\xi_{A}$ & Axial sweeping efficiency & {$[-]$} \\
\hline$\xi_{R}$ & Radial sweeping efficiency & {$[-]$} \\
\hline$\tau$ & $\omega t_{\text {pinch }}$ & \\
\hline$\langle\sigma\rangle\rangle$ & Thermonuclear cross section & {$\left[\mathrm{m}^{3} / \mathrm{s}\right]$} \\
\hline$\langle\sigma\rangle^{*}$ & Cross section coefficient, $3.3110^{-23} \mathrm{~m}^{3} / \mathrm{s}$ & {$\left[\mathrm{m}^{3} / \mathrm{s}\right]$} \\
\hline$\mu_{o}$ & Vacuum permeability, $4 \pi 10^{-7}$ & {$\left[\mathrm{~J} / \mathrm{mA}^{2}\right]$} \\
\hline$\omega$ & Ringing frequency & {$\left[\mathrm{s}^{-1}\right]$} \\
\hline
\end{tabular}

where the subscript $B$ refers to the Bennett equilibrium.
TABLE I

PARAMETERS USED IN THE MODEL

The effective thermonuclear cross section $\langle\sigma v\rangle$ is a function of the ions temperature according to

$$
\langle\sigma v\rangle=\langle\sigma v\rangle^{*} \theta^{-(2 / 3)} \exp \left(-\theta^{-(1 / 3)}\right)
$$

where $\theta=T / T^{*}$.

The characteristic time of the pinch $\Delta t$ is calculated as the half period of the harmonic oscillator associated with the linearization of (2) around the Bennett equilibrium, that is

$$
\ddot{r}+\left[\frac{h \mu_{o} I^{2}}{3 \pi M r_{B}^{2}}\right]\left(r-r_{B}\right)=0 .
$$

Taking into account (7) and (4), the pinch duration results:

$$
\Delta t=\frac{\pi r_{B}}{4} \sqrt{\frac{3 m_{i}}{k T_{B}}} .
$$

Combining (3)-(8) leads to

$\frac{Y}{h}=\frac{1}{128}\left(\frac{\mu_{o}}{4 \pi}\right)^{2} \sqrt{\frac{3 m_{i}\langle\sigma v\rangle^{* 2}}{\left(k T^{*}\right)^{5}}} I^{4} \frac{\theta_{B}^{-(19 / 6)} \exp \left(-\theta_{B}^{-(1 / 3)}\right)}{r_{B}}$. 


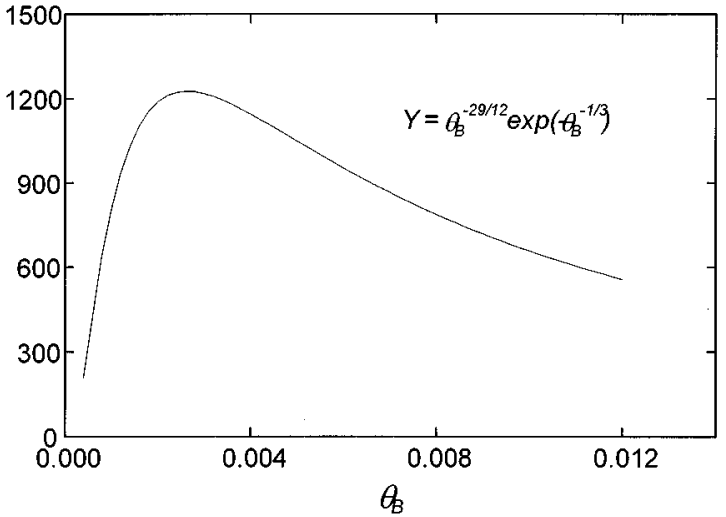

Fig. 3. Graphic of (11).

The Bennett radius $r_{B}$ is related to $\theta_{B}$ by means of the isoentropic hypothesis, that is

$$
r_{B}=r_{S} \theta_{B}^{-(3 / 4)}
$$

where $r_{S}$ is an appropriate isoentropic reference value.

Combining (9) and (10), $Y$ can be written in terms of only one variable $\theta_{B}$ :

$$
\frac{Y}{h}=\frac{1}{128}\left(\frac{\mu_{o}}{4 \pi}\right)^{2} \sqrt{\frac{3 m_{i}\langle\sigma v\rangle^{* 2}}{\left(k T^{*}\right)^{5}}} \frac{I^{4}}{r_{S}} \theta_{B}^{-(29 / 12)} \exp \left(-\theta_{B}^{-(1 / 3)}\right) .
$$

Equation (11) leads to a remarkable conclusion regarding the efficiency of thermonuclear pinches. There is a critical pinch temperature $\theta_{B}^{*}$ that produces the maximum neutron yield (see Fig. 3). Further efforts to increase the number of thermonuclear reactions by improving the pinch compression would be in vain once $\theta_{B}^{*}$ is reached, since higher pinch temperatures $\theta_{B}$ will reduce the efficiency. The maximum neutron yield occurs at $\theta_{B}^{*}=(4 / 29)^{3}$. The ideal optimum neutron yield corresponding to this maximum is given by

$$
\frac{Y_{o p t}}{h}=\frac{\sqrt{3}}{128 r_{S}}\left(\frac{29}{4 e}\right)^{29 / 4}\left(\frac{I}{I^{*}}\right)^{4}
$$

where the characteristic current $I^{*}$ is defined as

$$
I^{*}=\left[\left(\frac{4 \pi}{\mu_{o}}\right)^{4} \frac{\left(k T^{*}\right)^{\tilde{\Sigma}}}{m_{i}\langle\sigma v\rangle^{* 2}}\right]^{1 / 8} .
$$

The value of $I^{*}$ for deuterium plasma is about $88 \mathrm{kA}$.

\section{B. Dynamics of the Plasma Gun}

Consider now a PF device. As a first approximation, one can regard the final radial collapse as an ideal pinch, accepting (2) as valid in this stage. However, the current $I$ acting during the pinch is determined by the initial axial stage.

Let us assume a one-dimensional (1-D) lumped model of an annular current sheet, coupled with an external electric circuit whose main elements are a capacitor and some inductance. Considering an initial charge $Q_{o}$ in the capacitor and assuming an instantaneous ideal dielectric breakdown, the governing equations of the system are given by

$$
\begin{aligned}
& \frac{d}{d t}\left(m x \frac{d x}{d t}\right)=\frac{1}{2} l\left(\frac{d Q}{d t}\right)^{2} \\
& \frac{d}{d t}\left[(L+l x) \frac{d Q}{d t}\right]+\frac{Q}{C}=0 .
\end{aligned}
$$

The solutions to first order are

$$
\begin{aligned}
Q & =Q_{o} \cos \omega t \\
x^{2} & =\frac{l Q_{o}^{2}}{4 m}\left(t^{2} \omega^{2}-\sin ^{2} \omega t\right) .
\end{aligned}
$$

The optimum timing is achieved if the peak current $(\omega t=$ $\pi / 2)$ occurs simultaneously with the pinch $(x \approx z)$. Thus, the condition for the optimum timing is approximately

$$
z^{2}=\frac{l Q_{o}^{2}}{4 m}\left(\frac{\pi^{2}}{4}-1\right)
$$

If (17) is not satisfied, the current at the pinch would be less than $Q_{o} \omega$, and consequently, the neutron yield would not be optimum. Provided that $Q_{o}, l$, and $z$ are fixed, (17) can be tuned up by changing the initial deuterium pressure. In effect, $m$ and $p_{o}$ are related by

$$
m=\frac{\pi\left(r_{2}^{2}-r_{1}^{2}\right) \xi_{A} m_{i} p_{o}}{k T_{o}} .
$$

The current at the pinch $I$ for any given pressure $p_{o}$ is given by

$$
I=Q_{o} \omega \sin \omega t
$$

where $\omega t$ is related to $p_{o}$ by means of (16) and (18), with $x=z$.

\section{EXPERIMENTAL STUDY}

A PF pulsor prototype (GN1) was built in order to test the validity of the design model against reliable experimental data. The pulsor was designed to enhance the flux/fluence ratio by minimizing the size of the gas chamber. Fig. 4 shows the diagram of the experimental apparatus. The main parameters are given in Table II.

Series of measurements were conducted at several deuteriumfilling pressures in the range 2-7 mbar. The time derivative of the current flowing to the anode $d I / d t$ and the voltage across the electrodes $V$ were monitored for each shot by a Rogowski coil and a resistive voltage divider, respectively, and were registered using a $500-\mathrm{MHz}, 1-\mathrm{Gs} / \mathrm{s}$ digitizing oscilloscope. The time-integrated neutron yield was also measured for each shot by silver activation response. The neutron detector was placed at $60 \mathrm{~cm}$ from the focus in a line perpendicular to the axis. The neutron measuring system was calibrated by comparison against TLD detectors.

The timing from the current onset to the focusing time was measured from both the $d I / d t$ and $V$ waveforms. Fig. 5 shows 


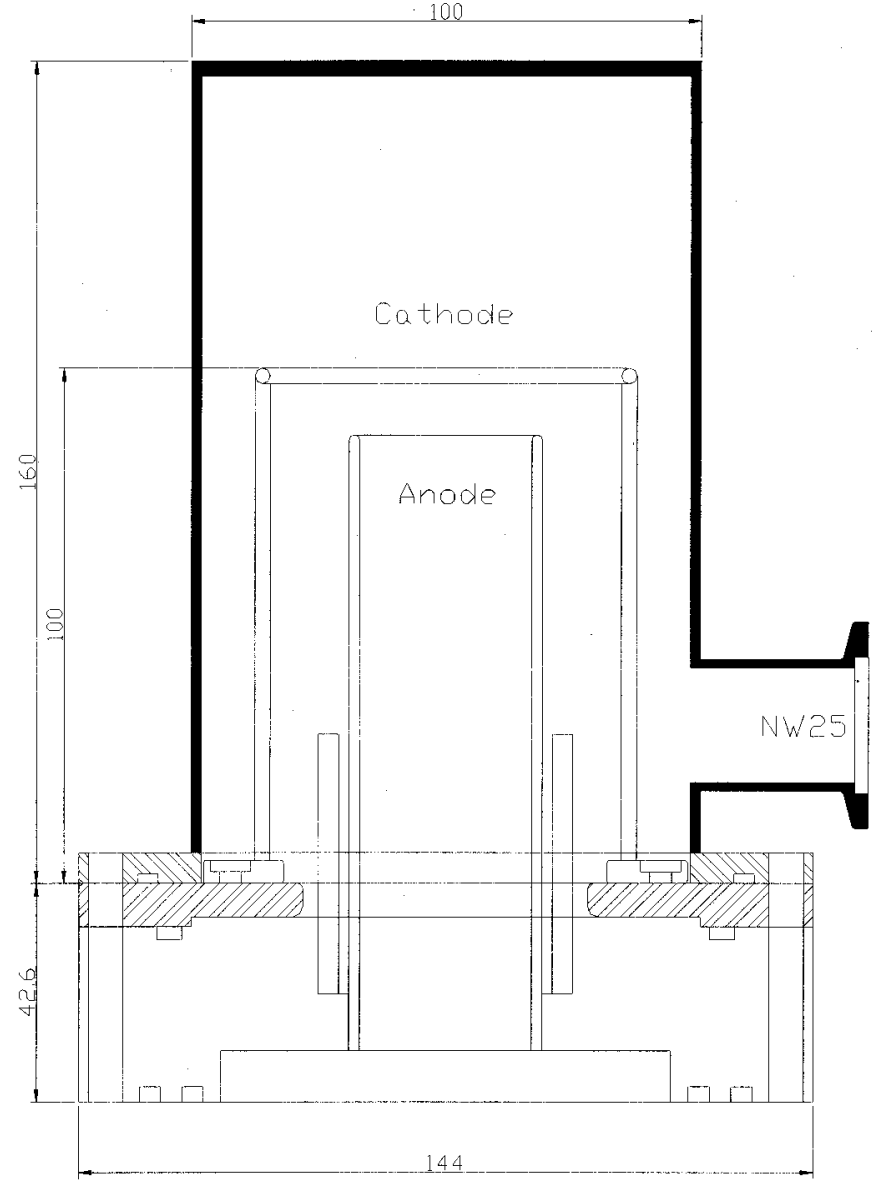

Fig. 4. Diagram of the prototype GN1.

TABLE II

PARAMETERS OF GN1

\begin{tabular}{l|c}
\hline Parameter & Value \\
\hline Capacity $[\mu \mathrm{F}]$ & 10.5 \\
Charging voltage $[\mathrm{kV}]$ & 30 \\
Stray inductance $[\mathrm{nH}]$ & 39 \\
Stored energy $[\mathrm{kJ}]$ & 4.725 \\
Anode radius [cm] & 1.9 \\
Cathode radius [cm] & 4.225 \\
Anode length [cm] & 8.71 \\
Cathode length $[\mathrm{cm}]$ & 10 \\
Insulator length [cm] & 3.5 \\
Insulator thickness [cm] & 0.4 \\
\hline
\end{tabular}

typical signals for filling pressures of 2, 4, and 6 mbar. Very intense voltage spikes are obtained at the time where the maximum compression takes place, thus indicating good focusing. It can also be observed that, as expected, the focus occurs later for higher pressures. Fig. 6 shows the average dependence of the focus timing with the deuterium pressure. The snow-plow theory presented in Section II-B corresponds to the curve shown in Fig. 6, showing excellent agreement with the experimental data.

Fig. 7 shows the dependence of the average neutron yield per pulse with the filling pressure. The curve corresponds to the model derived in Section II (4), (11), (16), (18), and (19) showing agreement within $20 \%$ of confidence.

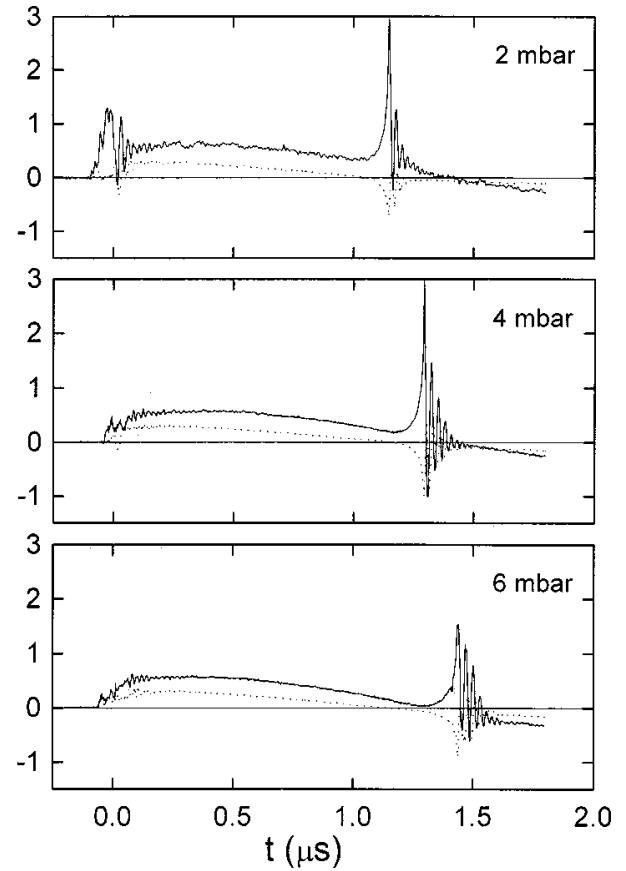

Fig. 5. Typical $d I / d t$ (dotted line) and $V$ (solid line) signals measured in prototype GN1 for several filling pressures.

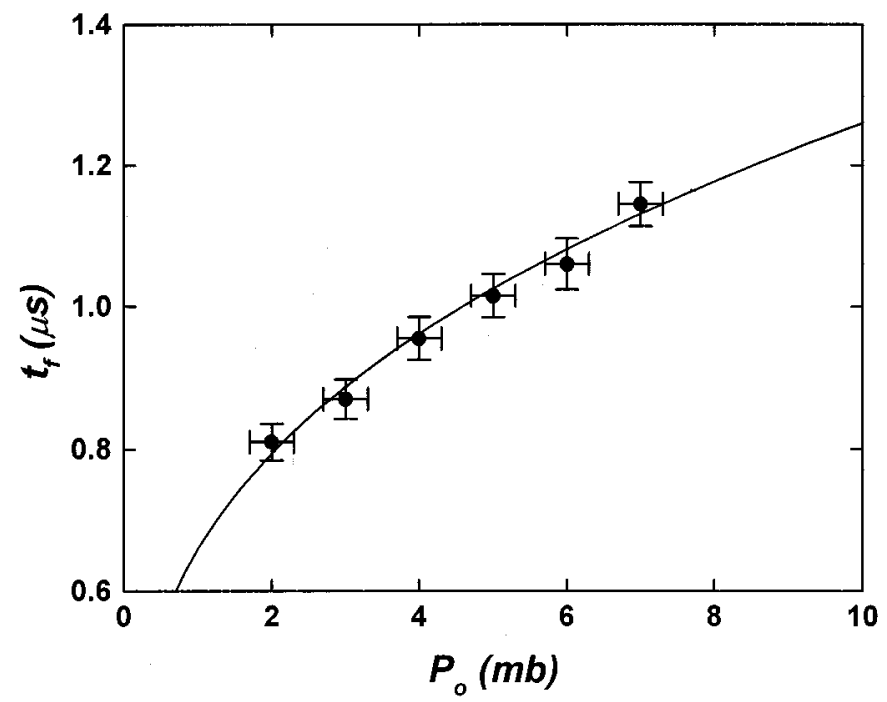

Fig. 6. Dependence of the focus timing with the deuterium filling pressure (GN1). $h=r_{1}, r_{s} / h=2.610^{-5}, \xi_{A}=0.17, \xi_{R}=0.07$.

\section{A. Benchmarking Against Other Experimental Data}

Two sets of experimental data from similar experiments reported elsewhere were compared with the theory, in order to assess the scope of the effective parameters. The data were selected from reports of low energy PF pulsors available in the open literature. Table III gives the main parameters of the experiments.

Figs. 8 and 9 show the dependence of the average neutron yield with filling pressure. The theory performs remarkably well using similar effective parameters.

Beg et al. [8] have carried out an interesting study that is very useful for the conceptual design of PF pulsors. The neutron yield dependence with pressure was studied using ten different 


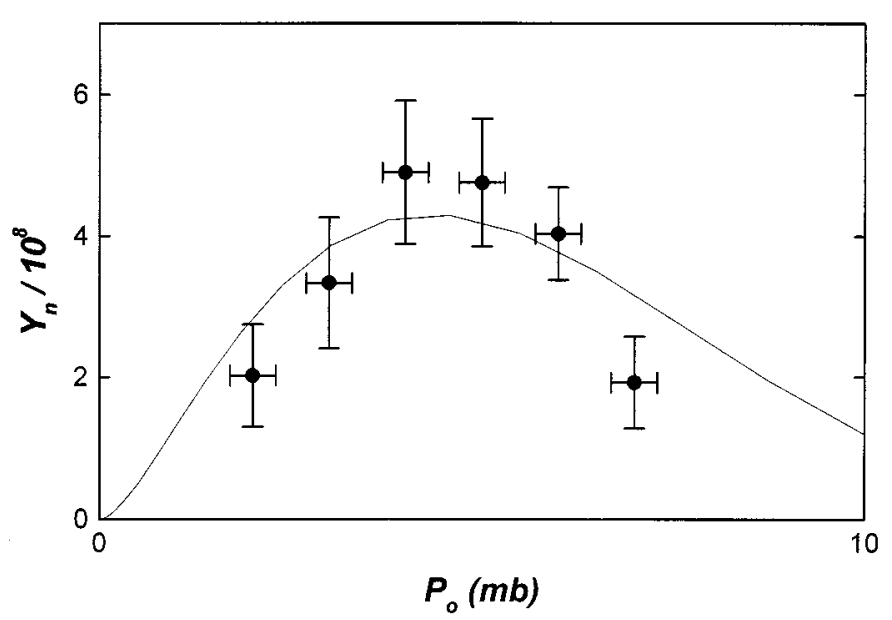

Fig. 7. Dependence of the average neutron yield with the deuterium filling pressure (GN1). $h=r_{1}, r_{s} / h=2.610^{-5}, \xi_{A}=0.17, \xi_{R}=0.07$.

TABLE III

PARAMETERS OF REPORTED DEVICES

\begin{tabular}{l|c|c}
\hline Parameter & Beg et al [8] & Nowikowski [9] \\
$\mathrm{z}[\mathrm{m}]$ & 0.148 & 0.23 \\
$\mathrm{r}_{1}[\mathrm{~m}]$ & 0.009 & 0.02 \\
$\mathrm{r}_{2}[\mathrm{~m}$ & 0.025 & 0.05 \\
$\mathrm{C}[\mu \mathrm{F}]$ & 32.7 & 21 \\
$\mathrm{~L}[\mathrm{nH}]$ & 80 & 75 \\
$\mathrm{~V}_{\mathrm{o}}[\mathrm{kV}]$ & 12 & 36 \\
\hline
\end{tabular}

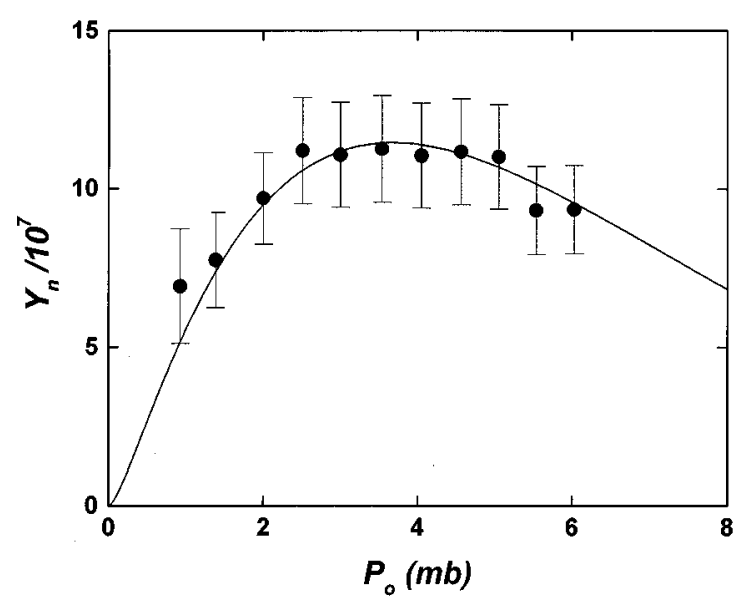

Fig. 8. Dependence of the average neutron yield with the deuterium filling pressure [8]. $h=r_{1}, r_{s} / h=6.110^{-6}, \xi_{A}=0.57, \xi_{R}=0.057$.

anode lengths. Figs. 10 and 11 show the maximum neutron yield and the corresponding optimum filling pressure for each anode length. It can be seen that the model is able to follow the experimental trend within the confidence bars.

Nowikowski [9] measured the average neutron yield changing the initial bank voltage at fix pressure. Fig. 12 shows the experimental data compared with the model, which again is able to follow the observed trend.

\section{OPTIMUM DESIGN CRITERION}

Equations (4) and (17) can be combined to derive an optimum design criterion for thermonuclear plasma focus devices. Equa-

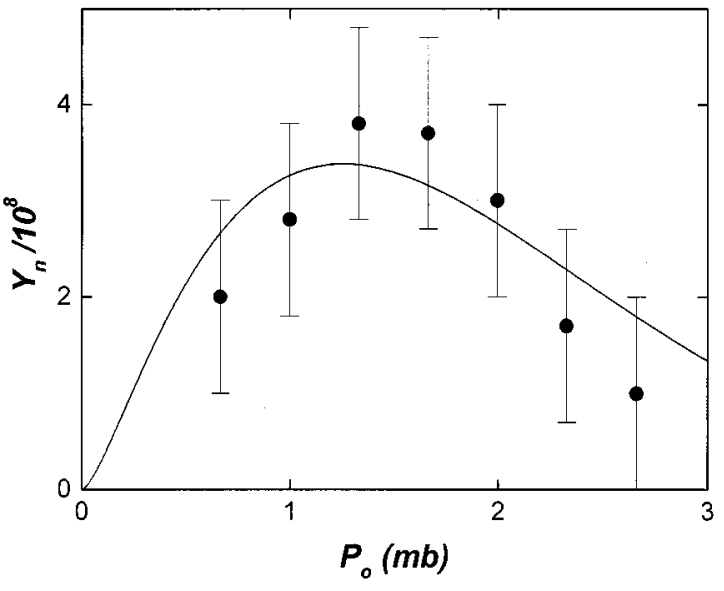

Fig. 9. Dependence of the average neutron yield $\left(90^{\circ}\right)$ with the deuterium filling pressure [9]. $h=r_{1}, r_{s} / h=510^{-5}, \xi_{A}=0.7, \xi_{A}=0.07$.

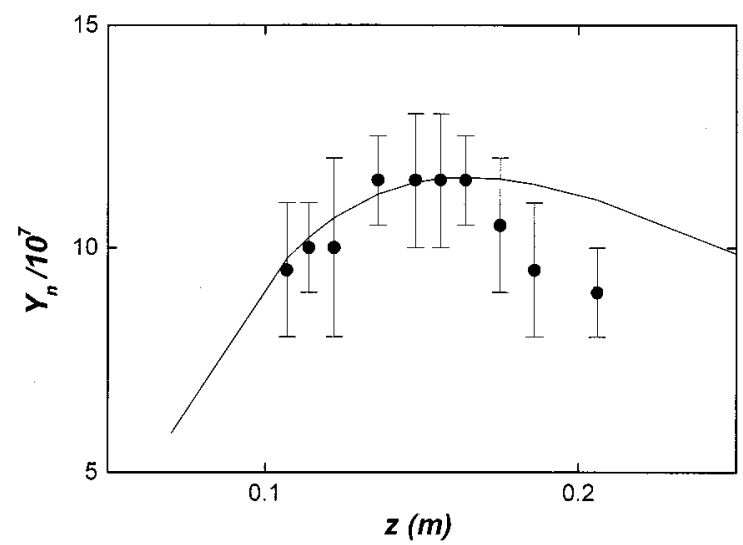

Fig. 10. Optimum neutron yield $\left(90^{\circ}\right)$ for different anode lengths [8]. $h=$ $r_{1}, r_{s} / h=6.110^{-6}, \xi_{A}=0.57, \xi_{R}=0.057$.

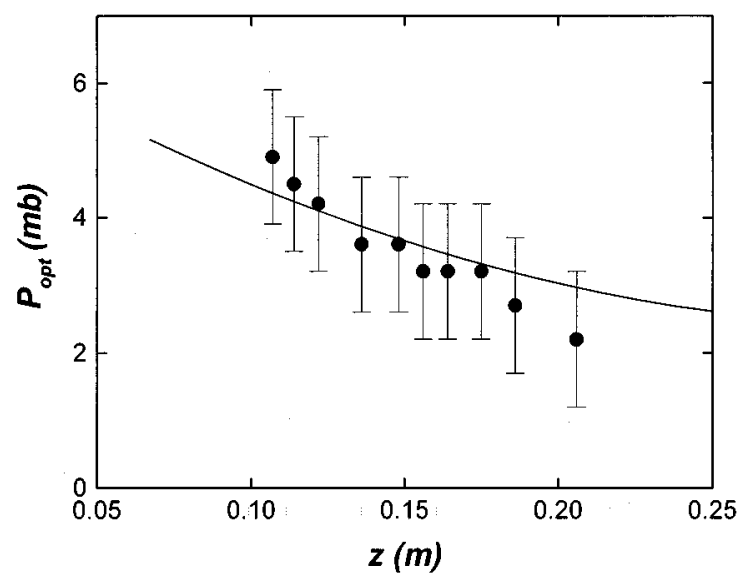

Fig. 11. Optimum filling pressure for different anode lengths [8]. $h=r_{1}, r_{s} / h=6.110^{-6}, \xi_{A}=0.57, \xi_{R}=0.057$.

tion (4) states that the number of deuterons per unit length that should be contained in the pinch to produce the optimum temperature $T_{B}^{*}$ is

$$
N=\frac{\mu_{\circ}}{16 \pi} \frac{I^{2}}{T_{B}^{*}} \frac{h}{k} .
$$




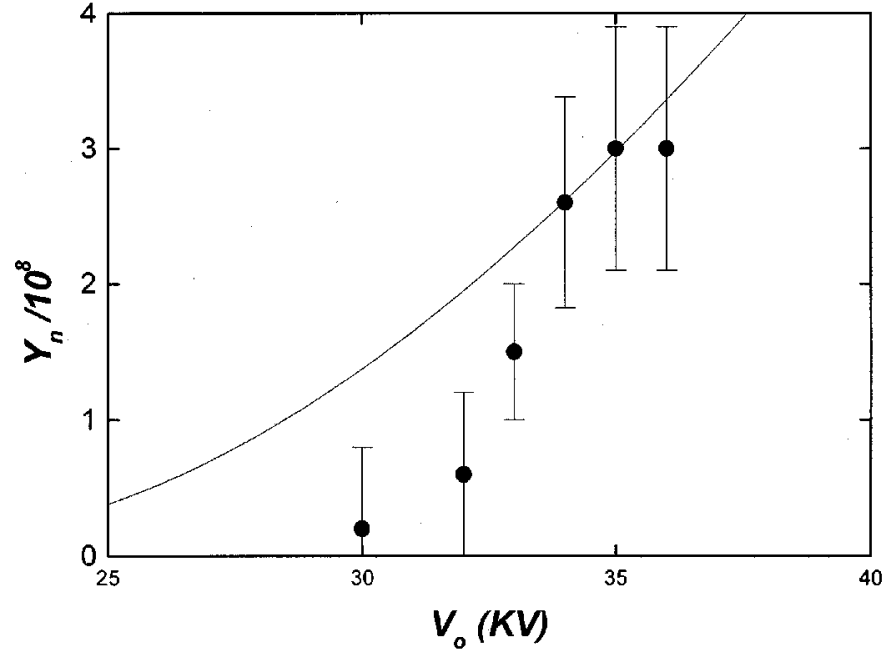

Fig. 12. Dependence of the neutron yield $\left(90^{\circ}\right)$ with the charge voltage [9]. $h=r_{1}, r_{s} / h=510^{-5}, \xi_{A}=0.7, \xi_{R}=0.07$.

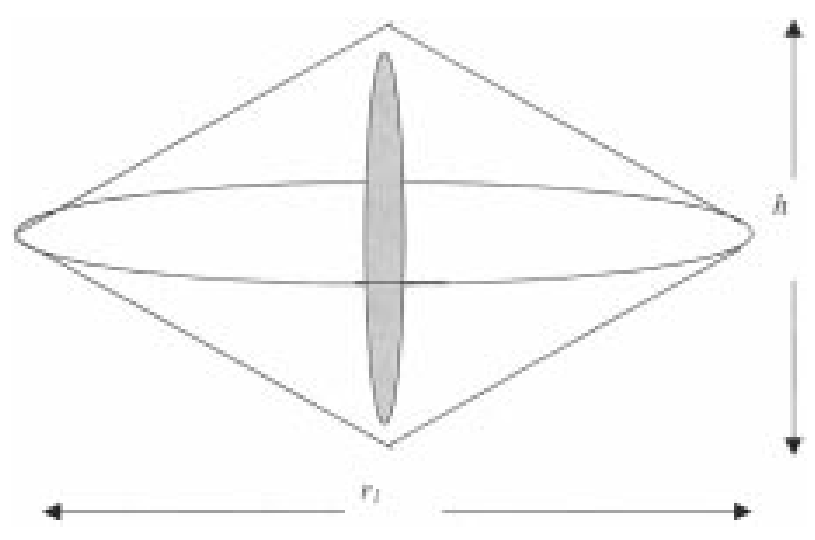

Fig. 13. Mass accumulated in the pinch.

On the other hand, the mass per unit length that should be collected in the axial region to induce the peak current at the pinch is, according to (17)

$$
m=\frac{l Q_{o}^{2}}{4 z^{2}}\left(\frac{\pi^{2}}{4}-1\right) .
$$

Both magnitudes in (20) and (21) are proportional to the filling gas density $\rho_{o}$. In particular

$$
m=\xi_{A} \rho_{o} \pi\left(r_{2}^{2}-r_{1}^{2}\right)
$$

and, assuming that the final pinch contains mostly the gas swept during the radial stage (see Fig. 13)

$$
N=\frac{\xi_{R} \rho_{o} h \pi r_{1}^{2}}{3 m_{i}} .
$$

Combining (20)-(23), the condition for the optimum design producing the peak current at the pinch and the optimum pinch temperature simultaneously is given by

$$
\lambda \omega=v^{*}
$$

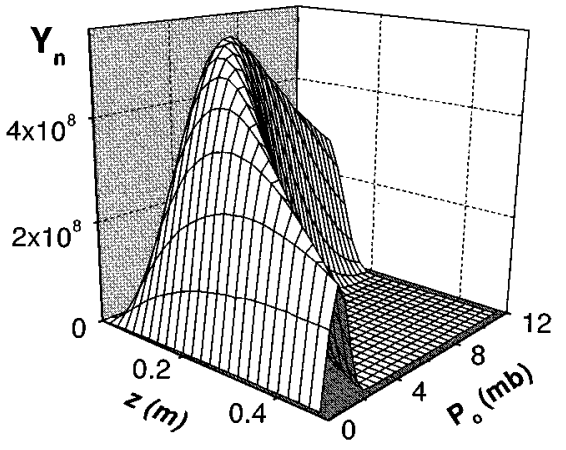

Fig. 14. Neutron yield dependence with the filling pressure and the electrode length, for the GN1 device.

where

$$
v^{*}=\sqrt{\frac{\left(\pi^{2} / 4-1\right)}{3} \frac{k T_{B}^{*}}{m_{i}}}
$$

and the characteristic length $\lambda$ is defined as

$$
\lambda=z \sqrt{\left(\frac{\xi_{A}}{\xi_{R}}\right) \frac{\left(r_{2} / r_{1}\right)^{2}-1}{\ln \left(r_{2} / r_{1}\right)^{2}}} .
$$

Fig. 14 shows the neutron yield dependence with the anode length and the filling pressure in GN1. The optimum yield is achieved when the peak current occurs simultaneously with the pinch $(\tau=\pi / 2)$ and the condition given by (24).

\section{CONCLUSION}

An analytical model of thermonuclear model of plasma focus devices is derived from the snowplow assumption and an isoentropic pinch oscillator. The model explains very well the filling pressure dependence data of three experiments performed in different devices. The results show that the hypothesis of thermonuclear reactions as the main source of the neutrons seems to be sufficient for obtaining most of the measured features of the neutron yield.

It is true that there is a significant literature, especially from Europe, that clearly shows the existence of beam-driven fusion reactions in the plasma focus. However, it is also clear that the GN1 device in this paper is being operated in the high pressure regime. Thus, a better comparison is with the Mather group literature from Los Alamos in the 1960s. That group clearly showed a scaling dependence of the fusion yield proportional to the current to the fourth.

A possible interpretation of what happened with the beams and beamlets inside the focus can be obtained by observing that the equilibrium times go as

$$
\tau_{i e} \propto \tau_{i i} \propto \frac{1}{I}
$$

where $\tau_{i e}$ and $\tau_{i i}$ stand for the ion-electron and ion-ion equilibrium times.

Therefore, as the current increases, beam contributions to the fusion yield via beam-hot target interactions become heating contributions to the core pinch. 
The supporting evidence presented in this paper does not preclude interpretation by other models, but the model set out here does at least facilitate the raising of hypothesis, and offers a simple conceptual design criterion of plasma focus devices.

\section{ACKNOWLEDGMENT}

The authors wish to thank two anonymous reviewers for their comments and observations that help to enhance the quality of the paper.

\section{REFERENCES}

[1] N. V. Filippov, T. I. Filippova, and V. P. Vinogradov, "High temperature dense plasma in a non-cylindrical $z$-pinch," Nucl. Fusion Suppl., vol. 2, p. 577, 1962.

[2] J. W. Mather, "Investigation of the high energy acceleration mode in the coaxial gun," Phys. Fluids Suppl., vol. 7, pp. 28-34, 1964.

[3] A. Bernard, P. Cloth, H. Conrads, A. Coudeville, C. Gourlan, A. Jolas, C. Maisonnier, and J. P. Rager, "The dense plasma focus: A high intensity neutron source," Nucl. Instr. Meth., vol. 145, pp. 191-218, 1977.

[4] H. Conrads, "Dense plasma focus as a neutron source for fusion research," Nucl. Sci. Eng., vol. 106, pp. 299-307, 1990.

[5] G. Decker and R. Wienecke, "Plasma focus devices," Physica, vol. 82C, pp. $155-164,1976$.

[6] D. E. Potter, "Numerical studies of the plasma focus," Phys. Fluids, vol 14, pp. 1911-1924, 1971.

[7] L. Bilbao, H. Bruzzone, and H. Kelly, "Structure of a plane and stationary ionizing current sheath," Plasma Phys. Contr. Fusion, vol. 26, pp. 1535-1548, 1984.

[8] F. N. Beg, M. Zakaullah, M. Nisar, and G. Murtaza, "Role of anode length in a Mather-type plasma focus," Modern Phys. Lett., vol. B6, pp. 593-597, 1992

[9] J. Nowikowski, "Preliminary investigations of a non-cylindrical z-pinch supplied from a $25 \mathrm{~kJ}$ current generator," Nukleonica, vol. 20, pp. 1081-1085, 1974
César Moreno received the M.Sc. and Ph.D. degrees in physics from the University of Buenos Aires, Buenos Aires, Argentina, in 1989 and 1995, respectively, working on Plasma Focus discharges.

Between 1995 and 1998, he was with the Colorado State University, Fort Collins, working in X-ray lasers. Since 1999, he has headed the neutron generators project of the Interinstitutional Program of Dense Plasmas, Buenos Aires. His current interests are with compact plasma focus and $z$-pinch devices and their applications.

Horacio Bruzzone received the M.Sc. and Ph.D. degrees in physics from the University of Buenos Aires, Buenos Aires, Argentina, in 1967 and 1976, respectively.

From 1981 to 1998, he was Associate Professor of Physics with the University of Buenos Aires and headed the experimental plasma physics group at the Institute of Plasma Physics, Buenos Aires. Since 1999, he has headed the Fast Pulsed Discharges Laboratory, University of Mar del Plata, Mar del Plata, Argentina. He is currently chairman of the scientific committee for the International Centre on Dense Magnetized Plasmas (Poland) and fellow of the National Science and Technology Council of Argentina (CONICET). His research interests are plasmas produced using fast pulsed discharges, with emphasis in Plasma Focus and $Z$-pinch devices.

Javier Martínez received the M.Sc. degree in physics in 1996 from the University of Buenos Aires, Buenos Aires, Argentina. He is currently FOMEC graduate student in the same university, majoring in physics of pulsed electrical discharges and their applications.

Alejandro Clausse received the nuclear engineering degree in 1981 and the Ph.D. degree in 1986 from Instituto Balseiro, Bariloche, Argentina.

$\mathrm{He}$ is Professor of Nuclear Engineering at the University of Cuyo, Cuyo, Argentina, and Professor of Computer Science at the University of the Center, Buenos Aires, Argentina. He was Visiting Scholar at the Rensselaer Polytechnic Institute, Troy, NY, from 1987 to 1990. Currently, he heads the PLADEMA Center of the Interinstitutional Program of Dense Plasmas, and the advanced reactors program of the National Atomic Energy Commission, Argentina.

Dr. Clausse is fellow of the National Science and Technology Council of Argentina (CONICET). 